\title{
BMJ Reducing postnatal depression, anxiety Open and stress using an infant sleep intervention
}

\author{
Brian Symon, Michael Bammann, Georgina Crichton, Chris Lowings, James Tucsok
}

To cite: Symon B, Bammann M, Crichton G, et al. Reducing postnatal depression, anxiety and stress using an infant sleep intervention. BMJ Open 2012;2:e001662.

doi:10.1136/bmjopen-2012001662

- Prepublication history for this paper are available online. To view these files please visit the journal online (http://dx.doi.org/10.1136/ bmjopen-2012-001662).

Received 13 June 2012 Accepted 15 August 2012

This final article is available for use under the terms of the Creative Commons Attribution Non-Commercial 2.0 Licence; see http://bmjopen.bmj.com
Kensington Park Medical Practice, Adelaide, Australia

\section{Correspondence to} Dr Brian Symon; brian.symon@adelaide.edu.au

\section{ABSTRACT \\ Objective: To examine the psychological well-being of mothers following participation in a behavioural modification programme previously shown to improve infant sleep.}

Design, setting and participants: A 45 min consultation with either a general practitioner (GP) or trained nurse providing verbal and written information describing sleep physiology in infants and strategies to improve infant sleep. Eighty mothers of infants 6-12 months of age with established infant sleep problems at a single general practice, Adelaide, South Australia.

Main outcome measures: The Depression Anxiety Stress Scale 21 (DASS21) immediately prior to the first consultation and again at follow-up approximately 3 weeks later. The number of infant nocturnal awakenings requiring parental support was also reported by mothers on both occasions.

Results: All measures of maternal well-being and infant nocturnal awakenings improved significantly. The mean number of maximum nocturnal awakenings decreased from 5.0 to 0.5 (mean difference 4.4, 95\% CI 3.4 to 5.5). All measures of DASS2 1 improved significantly. The mean total DASS21 decreased from 29.1 to 14.9 (mean decrease $14.2,95 \% \mathrm{Cl} 10.2$ to 18.2); mean depression decreased from 7.9 to 2.8 (mean difference $5.2,95 \% \mathrm{Cl} 3.7$ to 6.7 ); mean anxiety decreased from 4.6 to 2.6 (mean difference 2.0, 95\% $\mathrm{Cl} 0.7$ to 3.2); mean stress decreased from 16.6 to 9.5 (mean difference $7.0,95 \% \mathrm{Cl} 5.1$ to 9.0). The proportion of mothers assessed as having any degree of depression decreased by $85 \%$ from $26 / 80(32.5 \%)$ to $4 / 80(5 \%)$.

Conclusions: The number of nocturnal awakenings requiring parental support among infants aged $6-12$ months significantly decreased following a single consultation on infant sleep physiology and teaching behavioural strategies to improve sleep. Significant improvements in maternal stress, anxiety and depression were also observed.

\section{INTRODUCTION}

Approximately one-third of Australian parents report sleep difficulties in infants between the ages of 1-24 months. ${ }^{1}$ Between

\section{ARTICLE SUMMARY}

Article focus

- Postnatal depression is a common problem with significant morbidity.

- There is a known correlation with sleep deprivation.

- By improving infant sleep we have been able to show a reduction in maternal depression, anxiety and stress.

Key messages

- A behavioural sleep intervention in primary care can improve both infant sleep and maternal mental health.

Strengths and limitations of this study

- The intervention has been previously demonstrated in an randomised control trial.

- The study population was self-selected.

- The subjects were their own controls, before and after design.

the ages of $6-12$ months, this figure has been reported to be as high as $46 \%$. $^{2}$ Sleep problems in early infancy may have severe adverse consequences, including postnatal depression and maternal anxiety. It is estimated that $10 \%$ to $20 \%$ of women experience postnatal depression, ${ }^{3}$ while others experience emotional problems that while less severe than clinical depression may still negatively impact upon maternal wellbeing. ${ }^{2}{ }^{4-6}$ Maternal depression can have serious and long-term detrimental effects on family well-being and stability, and upon the cognitive and emotional development of the child. ${ }^{7-9}$ Furthermore, short sleep duration in early infancy can directly affect the child's behavioural competence, cognitive performance and physical development. ${ }^{5}$ Associations of parental reports of sleep problems with maternal depression have been previously demonstrated. $^{2} 10-12$

Patterns that can be characterised as sleep problems include a range of behaviours such as frequent and long nocturnal awakenings, being nursed to sleep, taking prolonged 
periods to fall asleep, sharing a bed with the parent and only taking short naps during the day. Behavioural interventions involving discussion of infant sleep and guidance on sleep improvement strategies have been previously demonstrated as an effective means of reducing infant sleep problems. ${ }^{13} 14$

In a previous randomised controlled trial, ${ }^{14}$ families were recruited at birth and randomly assigned to the control or intervention group. The intervention was a preventative strategy, a single $45 \mathrm{~min}$ consultation from a trained research nurse when the infant was aged between 2 and 3-weeks old. The consultation included a discussion on normal sleep patterns and the use of behavioural techniques to develop independent sleep skill'. Sleep diaries measured sleep performance at 6 and 12 weeks of age. There was significant sleep improvement observed in the intervention group at both follow-up points, including higher mean hours of day, night and total sleep without an increase in infant crying. At 6 weeks of age, infants in the intervention group averaged nearly $9 \mathrm{~h}$ more sleep per week and by 12 weeks this had increased to $14.6 \mathrm{~h} /$ week. At 12 weeks of age, $62 \%$ of the intervention group achieved at least $15 \mathrm{~h}$ mean sleep per 24-h period, compared with $36 \%$ of controls $(\mathrm{p}<0.001)$.

Compared with examining sleep outcomes, fewer studies have examined the effects of such sleep techniques on maternal mental health. ${ }^{15-17}$ This study applied the same intervention as our original trial ${ }^{14}$ to selfselected families experiencing sleep problems in infants aged between 6 and 12 months. The primary aims of the study were to examine reported infant nocturnal awakenings and depression, anxiety and stress in mothers after receiving this intervention. A secondary aim was to examine relationships between psychological outcomes and reported nocturnal awakenings. It is hypothesised that following implementation of the behavioural sleep intervention, the number of nocturnal awakenings and maternal depression, anxiety and stress will be significantly reduced.

\section{METHOD}

Study population and setting

Participants were mothers who presented to a metropolitan general medical practice in Adelaide, South Australia, and requesting assistance with infant sleep problems. The practice is a primary care site, which has specialised in the management of sleep problems in children. Patients who presented to the practice between June 2011 and October 2011 and who met the inclusion criteria were invited to participate in a study of maternal health and well-being by the primary researcher. Inclusion criteria were the child's age (6-12 months) and that the visit was the families first to the clinic. There were no other exclusion criteria. All participants gave informed written consent prior to completing the baseline questionnaire and receiving any treatment. Ethics approval was granted from the University of Adelaide Ethics Committee.

\section{Procedure and measures}

The Depression Anxiety Stress Scale 21 (DASS21) was completed by participants prior to seeing the clinician at their initial consultation. The DASS21 is a reliable, validated 21-item self-report measure designed to assess anxiety, depression and stress. ${ }^{18-20}$ Comprising three scales of seven items, each item is scored on a four-point scale ranging from 0 , 'did not apply to me at all', to 4 , 'applied to me very much, or most of the time'. The Depression scale assesses feelings of hopelessness, pessimism and lack of interest, involvement or enjoyment. The Anxiety scale assesses autonomic arousal, skeletal muscle effects such as shakiness, situational anxiety and performance worry. The Stress scale is sensitive to levels of nervous arousal, tension, ability to relax, irritability and ease of upset or agitation. Scores for Depression, Anxiety and Stress are calculated by summing the scores for each of the relevant items, and multiplying by 2 (to enable comparison with full DASS scores and clinical cut-offs). A severity rating is calculated for each component, ranging from 'normal' to 'extremely severe'. A follow-up DASS21 was completed before seeing the clinician at the second consult 2-3 weeks later, or by phone interview for those who did not return to the clinic. The number of typical nocturnal awakenings (minimum and maximum) was also reported by mothers at baseline and follow-up.

\section{Intervention}

The intervention comprised a 45-consultation provided by an experienced general practitioner (GP) with an expertise in infant sleep (BS) or a registered nurse (CL) trained to provide the same intervention working with the GP's supervision. Both parents were encouraged to attend. This intervention has been previously described, ${ }^{14}$ but included a discussion on normal sleep patterns in newborn infants (summarised in box 1) and the provision of written material reinforcing this information. The key points emphasised in the information provided were that sleep is cyclical with repeated bouts

Box 1 Key advice provided to families in consultation intervention

1. Human sleep is cyclical.

2. A return to sleep from an awakening is in part driven by external cues of sleep.

3. Cues of sleep are learned.

4. Sleep is usefully regarded as a learned skill.

5. Fatigue interferes with the performance of learned skills. In this context, fatigue impairs performing the skills of achieving and maintaining sleep.

6. Cues of sleep that are parent independent are the most useful. 
of waking, and that returning to sleep is driven by both fatigue and appropriate external 'cues of sleep' which are learned. Sleep achievement and maintenance are thus regarded as learned skills. Tiredness or more specifically over-tiredness is the enemy of high-quality sleep. Finally, it was emphasised that there is a need to avoid parent-dependent sleep cues as they lead to repeated cycles of 'requesting' or 'signalling' for parental support within a block of sleep. A 'cold turkey' approach was encouraged. A written copy of the tutorial was provided to each parent and they were encouraged to access a website (http://silentnights.org/) where a range of free supporting material was available.

\section{Statistical analysis}

A sample size of 33 was estimated to give $80 \%$ power to detect an effect size of 0.5 between before and after DASS scores, at an $\alpha$ of 0.05 . A nominal sample of 100 was chosen to allow for a drop-out rate of up to $50 \%$. Independent sample t tests were used to compare baseline demographic characteristics, and DASS21 scores between those who completed the study, and those who did not complete follow-up. Correlational analyses were used to examine any relationships between psychological outcomes and reported nocturnal awakenings. Paired $\mathrm{t}$ tests were used to determine any change in DASS21 scores (depression, anxiety, stress and total) between the two time points for each participant. All statistical analyses were conducted using SPSS for Windows, V.18.0 (SPSS Inc, Chicago, Illinois, USA).

\section{RESULTS}

\section{Participants}

In all, 103 mothers, aged 20-45 years, were invited to participate. Of which 99 were willing to participate, provided written consent and completed a baseline DASS21. Eighty $(81 \%)$ completed a follow-up DASS21 2-6 weeks later (median time to second measure was 25 days). Fifty-two mothers completed the follow-up DASS21 prior to their second consultation at the practice, and 28 completed the DASS21 by telephone. Of those who withdrew from the study $(n=19)$, two mothers declined to complete the follow-up questionnaire, and 17 were unable to be contacted and did not attend a second visit.

There was no significant difference in mother's age, child's age, depression, anxiety or stress scores or number of nocturnal awakenings at baseline for those who completed the study $(n=80)$, compared to those who withdrew $(n=19)$ (table 1). Families came from a wide variety of socio-economic demographics. The Socio-economic Index for Disadvantage was used to assess socio-economic status, and there was an even spread across all postcodes with no bias towards any advantage or disadvantage.

\section{Nocturnal awakenings}

At baseline, 12 (23\%) infants had five or more awakenings; $32(61 \%)$ had three or more nocturnal awakenings; $51(98 \%)$ had one or more awakening; and 1 (2\%) had no awakenings (table 2). At follow-up these figures had significantly improved, $0(0 \%)$ had five or more nocturnal awakenings; 2 (4\%) had three or more nocturnal awakenings; $16(31 \%)$ had one or more awakenings and $36(69 \%)$ had no awakenings.

Maternal stress was significantly and positively correlated with nocturnal awakenings at baseline and follow-up (both $\mathrm{p}<0.05$ ). Total DASS21 was significantly and positively correlated with nocturnal awakenings at follow-up $(\mathrm{p}<0.05)$.

\section{Depression, anxiety and stress}

There were significant improvements in Depression, Anxiety and total DASS21 (all $\mathrm{p}<0.001$ ), and for Stress $(\mathrm{p}=0.002)$ between baseline and follow-up (table 2$)$. Improvements were greatest in the following sequence: depression (66\% mean score reduction), total DASS21 (49\%), anxiety (44\%) and stress (42\%). Table 3 describes the distribution of DASS21 scores at baseline and follow-up, ranging from normal to extremely severe. Overall, $32.5 \%$ of mothers had depression categorised as mild or above at baseline, and at follow-up this figure was $5 \%$. Similarly, $48.8 \%$ and $25 \%$ of participants had mild or greater stress or anxiety respectively at baseline, which was reduced to $20 \%$ for stress and $15 \%$ for

\begin{tabular}{|c|c|c|c|}
\hline & \multicolumn{3}{|l|}{ Mean (SD) } \\
\hline & Completed $(\mathrm{n}=80)$ & Withdrawals $(n=19)$ & Total $(n=99)$ \\
\hline Maternal age at baseline (years) & $32.5(5.0)$ & $32.0(4.7)$ & $32.4(4.9)$ \\
\hline Child's age at baseline (months) & $8.5(1.7)$ & $8.4(2.3)$ & $8.4(1.8)$ \\
\hline Minimum number of nocturnal awakenings & $3.0(2.0)$ & $3.8(2.2)$ & $3.2(2.1)$ \\
\hline Maximum number of nocturnal awakenings & $4.4(3.3)$ & $5.4(4.1)$ & $4.6(3.4)$ \\
\hline Baseline depression & $7.9(8.3)$ & $6.2(4.2)$ & $7.6(7.7)$ \\
\hline Baseline anxiety & $4.6(6.3)$ & $4.2(6.0)$ & $4.5(6.2)$ \\
\hline Baseline stress & $16.6(9.4)$ & $16.8(11.4)$ & $16.6(9.8)$ \\
\hline Baseline DASS21 total & $29.1(21.1)$ & $27.3(20.0)$ & $28.7(20.8)$ \\
\hline
\end{tabular}


Table 2 Baseline, follow-up and change in DASS21 scores and number of nocturnal awakenings

\begin{tabular}{|c|c|c|c|c|c|c|}
\hline & \multirow[b]{2}{*}{$\mathbf{n}$} & \multirow{2}{*}{$\begin{array}{l}\text { Baseline } \\
\text { Mean (SD) }\end{array}$} & \multirow[t]{2}{*}{ Follow-up } & \multirow[b]{2}{*}{ Mean change $(95 \% \mathrm{Cl})$} & \multirow[b]{2}{*}{ Percentage change } & \multirow[b]{2}{*}{ p Value* } \\
\hline & & & & & & \\
\hline \multicolumn{7}{|l|}{ Primary outcomes } \\
\hline Depression & 80 & $7.9(8.3)$ & $2.8(4.7)$ & 5.2 (3.7 to 6.7$)$ & 65.8 & $<0.0001$ \\
\hline Anxiety & 80 & $4.6(6.3)$ & $2.6(4.6)$ & 2.0 (0.7 to 3.2$)$ & 43.5 & 0.002 \\
\hline Stress & 80 & $16.6(9.4)$ & $9.5(7.8)$ & 7.0 (5.1 to 9.0$)$ & 42.2 & $<0.0001$ \\
\hline DASS21 total & 80 & $29.1(21.1)$ & $14.9(15.8)$ & $14.2(10.2$ to 18.2$)$ & 48.8 & $<0.0001$ \\
\hline \multicolumn{7}{|l|}{ Secondary outcomes } \\
\hline Minimum number awakenings & 52 & $3.3(2.2)$ & $0.4(0.8)$ & 2.9 (2.2 to 3.5$)$ & 87.8 & $<0.0001$ \\
\hline Maximum number awakenings & 52 & $4.9(3.6)$ & $0.5(0.8)$ & 4.4 (3.4 to 5.5$)$ & 89.8 & $<0.0001$ \\
\hline
\end{tabular}

*Probability of differences between the two time points (paired sample t tests).

DASS, Depression, Anxiety, Stress Scale.

anxiety post-intervention. There were a total of 27 severe or extremely severe scores in depression, anxiety or stress at baseline. This was reduced to seven scores at follow-up.

\section{DISCUSSION}

It has previously been demonstrated that a single consultation provided in a primary health-care setting can improve infant sleep by up to $14.6 \mathrm{~h} /$ week. $^{14}$ The application of this strategy to families with established sleep problems has demonstrated a clear improvement in infant sleep and importantly, significant improvement in the psychological well-being of mothers. There was a significant reduction in maternal depression, anxiety and

Table 3 Distribution of depression, anxiety and depression scores at baseline and follow-up, ranging from normal to extremely severe for $n=80$

\begin{tabular}{lcc}
\hline & Baseline & Follow-up \\
\cline { 2 - 3 } & $\mathbf{n}(\%)$ & \\
\hline Normal & & \\
$\quad$ Depression 0-9 & $54(67.5)$ & $76(95)$ \\
Anxiety 0-7 & $60(75.0)$ & $68(85)$ \\
Stress 0-14 & $41(51.2)$ & $64(80)$ \\
Mild & & \\
Depression 10-13 & $13(16.3)$ & 0 \\
Anxiety 8-9 & $6(7.5)$ & $5(6.3)$ \\
Stress 15-18 & $12(15.0)$ & $10(12.5)$ \\
Moderate & & \\
$\quad$ Depression 14-20 & $6(7.5)$ & $3(3.8)$ \\
Anxiety 10-14 & $9(11.3)$ & $5(6.3)$ \\
Stress 19-25 & $12(15.0)$ & $2(2.5)$ \\
Severe & & \\
Depression 21-27 & $3(3.8)$ & 0 \\
Anxiety 15-19 & $1(1.3)$ & 0 \\
Stress 26-33 & $10(12.5)$ & $2(2.5)$ \\
Extremely severe & & \\
Depression 28+ & $4(5.0)$ & $1(1.3)$ \\
Anxiety 20+ & $4(5.0)$ & $2(2.5)$ \\
Stress 34+ & $5(6.3)$ & $2(2.5)$ \\
\hline
\end{tabular}

stress scores (all $\mathrm{p}<0.003)$ following one single sleep behaviour consultation. Importantly, the changes observed reflect a clinically and socially significant improvement in outcome scores. A third of mothers $(32.5 \%)$ reported at least mild levels of depression at baseline, and at follow-up this figure had decreased to $5 \%$, with similar results for anxiety and stress. Most importantly, the numbers of women in the severe/ extremely severe categories at baseline were all reduced at the post-intervention assessment.

Our findings add support to previous studies. Three randomised controlled trials have demonstrated that sleep management programmes can have a beneficial effect on reducing depression prevalence, and improve sleep performance. ${ }^{15-17}$ Armstrong et $a l^{15}$ implemented an outpatient-based sleep management programme conducted over a longer time frame, and with older children than in the present study. Depression was reduced from $40 \%$ of the sample at baseline, to $4.3 \%$ at an average of 2 months later. Similarly, this study sample consisted of parents presenting to a sleep clinic and the intervention was the provision of 'controlled crying' and 'cold turkey' techniques. Significant improvements in sleep parameters also occurred. Controlled crying techniques require the parent to put the baby down awake at sleep times, and allow him to go to sleep with minimal intervention. The infant may cry for a certain time, and if not settled to sleep by this time, parental reassurance is required. This response may involve a check of the infant, a rewrap, or perhaps a short top-up feed, and then returning the infant to attempt to sleep again. This parental response to crying occurs at increasing time intervals.

A larger randomised trial by Hiscock $e t a l^{16} 17$ involved over 300 mothers reporting an infant sleep problem at 7 months of age. The intervention comprised consultations regarding behavioural strategies, the formation of a sleep management plan and handouts on normal sleep patterns. The behavioural intervention consisted of two options: (1) 'controlled crying' whereby the parent responded to infant crying at increasing intervals, or (2) 'camping out' whereby the parent sat with the 
infant until they fell asleep, gradually removing their presence over 3 weeks. ${ }^{16}{ }^{17}$ Depression was significantly lower at both 10 and 12 months for the intervention mothers compared with controls, as was the reporting of sleep problems. These improvements appear to have been maintained at 2 years, with intervention mothers having $59 \%$ lower odds of reporting depressive symptoms at this time. ${ }^{17}$

The significant morbidities associated with problems of infant sleep have been reported extensively, particularly postnatal depression. A large meta-analysis of 59 studies, including over 12000 women and using a range of different assessment methods, estimated that overall prevalence of postpartum depression was $13 \% .^{3}$ Australian studies have reported figures ranging from $6.2 \%^{21}$ to $7.5 \%,{ }^{22}$ with figures increasing to $22.6 \%$ when a lower cut-off (for minor depression) is used. ${ }^{23}$ These studies were all conducted within the first 2 months of the infant's life. Prevalence at longer follow-ups between 5 and 9 months postpartum have shown higher prevalence's of between $13.9 \%$ and $16.9 \%$ for major depression, ${ }^{24}$ and $18.2 \%$ for minor depression. ${ }^{25}$ Maternal anxiety, given much less attention than postnatal depression, may also be experienced by a large number of women. Panic attacks or intense anxiety has been reported by up to $15.7 \%$ in the first 3 months postpartum, and $8.5 \%$ from 6 to 9 months postpartum in Australian mothers. ${ }^{25}$ Based on the number of registered births in Australia in 2010 (nearly 300 000), and using a prevalence estimate of $15 \%$, a possible 45000 women per year may experience some degree of postnatal depression, clearly an enormous public health problem. The high rates of maternal anxiety and depression in mothers of infants with sleep problems have been previously demonstrated. ${ }^{2}{ }^{10-12}$ Assisting these mothers to improve their infant's sleep is therefore a very important factor in reducing depression. An effective behavioural strategy, such as the one presented, implemented by parents in the home environment to significantly improve infant sleep could potentially have a significant impact on reducing the rates of postnatal depression in Australian mothers.

It is well established that the detrimental impacts of postpartum depression extend beyond the parent to the child. Deficits in early interactions and cognitive functioning, insecure infant attachment and behavioural disturbances have been associated with postpartum depression. ${ }^{8}$ Second, the lack of prolonged sleep itself, can have detrimental effects on child development, including behavioural, cognitive and physical parameters. ${ }^{5}$ An effective intervention, which can improve sleep behaviour in infants, and subsequently symptoms of depression in mothers, may therefore benefit the mother and the child. This is a simple consultation-based strategy, that is, time effective, and can be readily taught to a healthcare professional in a primary care setting. It has the potential to be an extremely cost-effective means of improving infant sleep and maternal mental health.
Some limitations must be acknowledged. Our recruitment methods relied upon women presenting to a local medical practitioner for assistance. These women had therefore identified a problem and been willing to seek help. Women aged under 20, and of non-Englishspeaking background were likely to have been unrepresented in the study. Data were not collected on other socio-demographic determinants such as family income, education or relationship status. Without this information or comparative population data, the representativeness of the sample cannot be concluded.

The definition of a sleep problem, in the absence of an accepted form of measurement, was defined according to parental report. However, the collection of the number of nocturnal awakenings confirmed that these infants did have broken sleep. We are unable to define the direction of causality between sleep problems and maternal mental health. The intervention employed in the current study, effective in improving infant sleep behaviour, is concurrently targeting psychological well-being.

Information regarding the number of nocturnal awakenings was not obtained from those women who completed the follow-up by telephone $(n=28)$, which is a shortcoming of the study. Measures of maternal sleep quality and quantity, or other measures of maternal health apart from the psychological measures were not obtained. It also must be acknowledged that it is possible that participants were receiving other treatment or assistance for either their depression or their infant's sleep problems during the study period. A major limitation of the before-and-after study design is the inability to control for other external factors that may impact upon the outcomes. Other external factors that may have impacted upon the results, and that were not measured, include family or social stressors. Subsequently, any cause-and-effect conclusions cannot be drawn. Finally, we cannot determine whether non-respondents to the second questionnaire did not have intervention success and therefore were not willing to complete the questionnaire. Despite these limitations, the findings are suggestive that this behavioural technique may be of benefit to mothers experiencing some degree of postnatal depression, anxiety or stress. The intervention may be quite easily replicated by trained physicians or nurses in other primary healthcare settings, particularly in clinical practices that specialise in infant and maternal health.

\section{Conclusions}

Our findings indicate that effectively managing infant sleep problems may be a key component of treating postnatal depression. The intervention promotes the development of good sleep habits by putting the infant to sleep awake and without parental intervention. This behavioural intervention may potentially present as a timely and effective technique for reducing postnatal depression, anxiety and stress in mothers. With the adverse impact that both depression and sleep deprivation have on mother and child, the beneficial implications of this 
intervention for families are immense. Randomised controlled trials are needed to confirm these positive findings, and to examine the impact of the behavioural intervention on infant sleep performance and maternal health, including psychological outcomes and sleep, in the long term.

Contributors BS, JT and MB developed the study design. MB collected patient data. $C L$ and $B S$ delivered the intervention. $M L, G C$ and $M B$ analysed data. GC, BS and MB drafted the manuscript. All authors read and approved the final manuscript.

Competing interests None.

Provenance and peer review Not commissioned; externally peer reviewed.

Data sharing statement Requests for access to data should be made to the corresponding author (brian.symon@adelaide.edu.au).

\section{REFERENCES}

1. Armstrong KL, Quinn RA, Dadds MR. The sleep patterns of normal children. Med J Aust 1994;161:202-6.

2. Hiscock $H$, Wake $M$. Infant sleep problems and postnatal depression: a community-based study. Pediatrics 2001;107:1317-22.

3. Ohara MW, Swain AM. Rates and risk of postpartum depression-a meta-analysis. Int Rev Psychiatry 1996;8:37-54.

4. Brown S, Lumley J. Maternal health after childbirth: results of an Australian population based survey. Br J Obstet Gynaecol 1998:105:156-61.

5. Touchette E, Petit D, Tremblay RE, et al. Risk factors and consequences of early childhood dyssomnias: new perspectives. Sleep Med Rev 2009;13:355-61.

6. Yelland J, Sutherland G, Brown SJ. Postpartum anxiety, depression and social health: findings from a population-based survey of Australian women. BMC Public Health 2010;10:771.

7. Beck CT. The effects of postpartum depression on child development: a meta-analysis. Arch Psychiatr Nurs 1998;12:12-20.

8. Murray L, Cooper PJ. The impact of postpartum depression on child development. Int Rev Psychiatry 1996;8:55-63.

9. Cogill SR, Caplan HL, Alexandra $\mathrm{H}$, et al. Impact of maternal postnatal depression on cognitive development of young children. BMJ 1986;292:1165-7.

10. Lam P, Hiscock H, Wake M. Outcomes of infant sleep problems: a longitudinal study of sleep, behavior, and maternal well-being. Pediatrics 2003;111:e203-7.
11. Zuckerman B, Stevenson J, Bailey V. Sleep problems in early childhood-continuities, predictive factors, and behavioral correlates. Pediatrics 1987;80:664-71.

12. Armstrong $\mathrm{KL}$, O'Donnell $\mathrm{H}$, McCallum $\mathrm{R}$, et al. Childhood sleep problems: association with prenatal factors and maternal distress/ depression. J Paediatr Child Health 1998:34:263-6.

13. Hiscock H, Wake M. Randomised controlled trial of behavioura infant sleep intervention to improve infant sleep and maternal mood. BMJ 2002;324:1062-5.

14. Symon BG, Marley JE, Martin AJ, et al. Effect of a consultation teaching behaviour modification on sleep performance in infants: a randomised controlled trial. Med J Aust 2005;182:215-18.

15. Armstrong KL, Van Haeringen AR, Dadds MR, et al. Sleep deprivation or postnatal depression in later infancy: separating the chicken from the egg. J Paediatr Child Health 1998;34:260-2.

16. Hiscock H, Bayer J, Gold L, et al. Improving infant sleep and maternal mental health: a cluster randomised trial. Arch Dis Child 2007;92:952-8.

17. Hiscock H, Bayer JK, Hampton A, et al. Long-term mother and child mental health effects of a population-based infant sleep intervention: cluster-randomized, controlled trial. Pediatrics 2008; 122:E621-7.

18. Crawford JR, Henry JD. The Depression Anxiety Stress Scales (DASS): normative data and latent structure in a large non-clinical sample. Br J Clin Psychol 2003;42:111-31.

19. Lovibond PF, Lovibond SH. The structure of negative emotional states: comparison of the Depression Anxiety Stress Scales (DASS) with the Beck depression and anxiety inventories. Behav Res Ther 1995;33:335-43.

20. Lovibond SH, Lovibond PF. Manual for the depression anxiety stress scales. 2nd edn. Sydney: Psychology Foundation, 1995.

21. Eastwood JG, Phung H, Barnett B. Postnatal depression and socio-demographic risk: factors associated with Edinburgh Depression Scale scores in a metropolitan area of New South Wales, Australia. Aust N Z J Psychiatry 2011;45:1040-6.

22. Buist AE, Austin MPV, Hayes BA, et al. Postnatal mental health of women giving birth in Australia 2002-2004: findings from the beyondblue National Postnatal Depression Program. Aust N Z J Psychiatry 2008;42:66-73.

23. Edwards B, Galletly C, Semmler-Booth $\mathrm{T}$, et al. Does antenatal screening for psychosocial risk factors predict postnatal depression? A follow-up study of 154 women in Adelaide, South Australia. Aust N Z J Psychiatry 2008;42:51-5.

24. Brown S, Bruinsma F, Darcy MA, et al. Early discharge: no evidence of adverse outcomes in three consecutive population-based Australian surveys of recent mothers, conducted in 1989, 1994 and 2000. Paediatr Perinat Epidemiol 2004;18:202-13.

25. Woolhouse H, Brown S, Krastev A, et al. Seeking help for anxiety and depression after childbirth: results of the Maternal Health Study. Arch Women Ment Health 2009;12:75-83. 